report by Ronen and associates stresses the unfavorable prognosis of neonatal seizures, especially in preterm infants, and with the exception of clonic seizures without facial involvement. Outcome is determined primarily by gestational age and the severity and timing of the encephalopathy resulting in seizures. The authors emphasize the need for preventive measures and antiepileptic medications more specific for neonatal seizures.

A study of the etiology and neurodevelopmental outcome of seizures in term newborn infants (Tekgul H et al. Pediatrics 2006;117:1270-1280; Ped Neur Briefs April 2006;20:2930 ) found that neonatal mortality was $7 \%$, and neurologic outcome of survivors at 1 year was favorable in $72 \%$. Predictors of a favorable outcome were a normal neonatal neurologic exam and normal EEG. Global cerebral hypoxic-ischemia is the most frequent cause $(40 \%)$ of neonatal seizures and a strong predictor of poor long-term outcome. The evidence favors the etiology as the most important factor in prognosis and not the neonatal seizure per se. (Camfield PR. Epilepsia 1997;38:735-737). In developing laboratory animals, a major tonicclonic seizure with post-ictal depression cannot be induced until the animal is older and cortical organization more mature. (Millichap JG. Proc Soc Exper Biol \& Med 1957;96:125-129).

\title{
PAROXYSMAL NONEPILEPTIC EVENTS
}

Paroxysmal events that mimic epilepsy, and their precipitants, prodromes, and distinguishing features are reviewed by researchers at Texas Tech University, Lubbock, TX, and American University of Beirut, New York. They include syncope, long QT disorders, breath-holding episodes, compulsive self-induced valsalva syncopal convulsions, apneic episodes, hyperekplexia, familial hemiplegic migraine, basilar migraine syncope, benign paroxysmal vertigo, alternating hemiplegia, Alice in Wonderland syndrome, psychogenic seizures, motor tics, paroxysmal torticollis, paroxysmal dyskinesias, dystonic choreoathetosis, kinesigenic choreathetosis, paroxysmal dystonia induced by exercise, episodic ataxia, benign myoclonus of early infancy, shuddering attacks or tremors, neonatal jitteriness, Sandifer syndrome (gastroesophageal reflux), stereotypies (repetitive movements), paroxysmal tonic upward gaze, oculomotor apraxia, spasmus nutans, opsoclonus, daydreaming, sleep disorders, nonrapid eye movement arousal disorders, rapid eye movement nightmares or sleep paralysis, sleep transition head banging and body rocking, benign neonatal sleep myoclonus, and narcolepsy-cataplexy syndrome. These potential mimickers of epilepsy may be classified according to age of occurrence and clinical presentation. (Obeid M, Mikati MA. Expanding spectrum of paroxysmal events in children: potential mimickers of epilepsy. Pediatr Neurol Nove 2007;37:309-316). (Dr Mikati, Epilepsy Program, Department of Pediatrics, American University of Beirut, $8503^{\text {rd }}$ Ave, $18^{\text {th }}$ Floor, New York, NY 10020).

COMMENT. As presented in this extensive review, paroxysmal nonepileptic events in infants and children comprise an expanding spectrum. The misdiagnosis of epilepsy in children with apparent refractory seizures, reported to be as high as $39 \%$ (Uldall P et al. Arch Dis Child 2006;91:219-221; cited by reviewers), leads to unnecessary investigation and potentially toxic antiepileptic therapy. Diagnosis of a nonepileptic event requires a careful history and a normal ictal EEG. 